\title{
Analytical determination of Yukawa Potential: An iterative semiclassical approach
}

\author{
Breno Rodrigues Segatto*1@ \\ ${ }^{1}$ Universidade Federal do Espírito Santo, Departamento de Física, Vitória, ES, Brasil.
}

Received on January 07, 2020. Revised on April 08, 2020. Accepted on April 27, 2020.

\begin{abstract}
In this work we propose an alternative semiclassical iterative approach to obtain the Yukawa Potential, where the temporal evolution is replaced by the number of iterations. In addition, our analytical approach was able to provide an exact value very close to the adopted semi-empirically for the Yukawa magnitude scale constant.

Keywords: Yukawa Potential, analytical model, meson.
\end{abstract}

\section{Introduction}

Yukawa in the first half of the $20^{t h}$ century proposed that the nucleons were bound in the nucleus through an interaction that was mediated by mesons, [1] this hypothesis was later confirmed by Lattes et al. 2] This interaction is short-range and its potential was originally derived as being

$$
U=-\frac{g^{2}}{r} e^{-\mu r},
$$

where $g^{2}$ is a magnitude scaling constant and e $1 / \mu$ is the range of the potential.

Short-range potentials of type

$$
U=-A f(r) e^{-\mu r},
$$

(where $f(r)$ is a function that depends on the spacial dimensions) have already been studied before Yukawa 1 in the most varied areas, 3] namely: celestial mechanics [4], electrostatics [5], inter-molecular interactions [6], fluid theory [7] and electrolysis [8, 9].

Due to their importance, appearance in diverse physical systems and due to still remains an object of study 10 16, we have decided to propose an alternative approach to obtain this short-range potential.

\section{Iterative Approach}

Let us consider here a semiclassical system and consider the relative motion of a test particle of mass $m$ in a two-body system. At an initial time $t_{0}$ its position and momentum are $r_{0}$ and $p_{0}$ respectively. After the first iteration

$$
t_{1}=t_{0}+\delta t_{0}
$$

${ }^{*}$ Correspondence email address: breno.segatto@ufes.br

$$
r_{1}=r_{0}+\delta r_{0}
$$

and

$$
p_{1}=p_{0}+\delta p_{0} .
$$

where $\delta t, \delta x$ and $\delta p$ are the increments of their respective variables.

The system again follows a straight trajectory until its second iteration at the instant

$$
t_{2}=t_{1}+\delta t_{1},
$$

which changes its momentum to

$$
p_{2}=p_{1}+\delta p_{1}
$$

in position

$$
r_{2}=r_{1}+\delta r_{1} .
$$

Thus, after the $\mathrm{n} t h$ iteration

$$
t_{n}=t_{n-1}+\delta t_{n-1}=t_{0}+\sum_{j=0}^{n-1} \delta t_{j},
$$

$$
p_{n}=p_{n-1}+\delta p_{n-1}=p_{0}+\sum_{j=0}^{n-1} \delta p_{j}
$$

and

$$
\begin{aligned}
r_{n} & =r_{n-1}+\delta r_{n-1}=r_{0}+\sum_{j=0}^{n-1} \delta r_{j} \\
& =r_{0}+\sum_{j=0}^{n-1} \frac{p_{j}}{m} \delta t_{j} .
\end{aligned}
$$


In the equation 11 we use the relation

$$
\delta r_{k}=\frac{p_{k-1}}{m} \delta t_{k-1} .
$$

The equations (9), 10) and (11) are generic and valid for any semiclassical physical system.

\section{Obtaining the Yukawa Potential}

Let us consider a law of force proportional to the inverse of the square of the distance between two particles, i.e. like Newton/Coulomb's Law

$$
\delta p_{j}=\sigma \frac{\delta t_{j-1}}{r_{j-1}^{2}}
$$

where $\sigma$ is a constant of proportionality.

Let us consider that the field-generating particle (gravitational or electric) is at the origin of the considered coordinate system and that the test particle is in the position $r_{0}$ with momentum $p_{0}$ and that $r_{0}<1$ with $\delta r_{j}<<1$, this ensures that the two particles are very close to each other (such as inside a nucleus). We will also consider $\delta t_{j}<<1$, this ensures that the iterative process approaches the continuous fields picture as expected, it is important to note that there are analytic approaches in which the fields are considered as discrete, [17-19] is not the case in question. Defining the time increment as being constant, i.e., $\delta t_{j} \equiv \omega>0$, equations (11) and 10 then become

$$
r_{n}=r_{0}+\frac{\omega}{m} \sum_{j=0}^{n-1} p_{j}
$$

and

$$
\begin{aligned}
p_{n} & =p_{0}+\sigma \omega \sum_{j_{0}=0}^{n-1} \frac{1}{r_{j_{0}-1}^{2}} \\
& =p_{0}+\sigma \omega \sum_{j_{0}=0}^{n-1}\left(r_{0}+\frac{\omega}{m} \sum_{j_{1}=0}^{n-1} p_{j-1}\right)^{-2} \\
& =p_{0}+\frac{\sigma \omega}{r_{0}^{2}} \sum_{j_{0}=0}^{n-1}\left(1+\frac{\omega}{m r_{0}} \sum_{j_{1}=0}^{j_{0}-1} p_{j-1}\right)^{-2}
\end{aligned}
$$

respectively. The last term of equation (15) refers to the increase in position which is much smaller than one, consequently we can rewrite equation 15 as:

$$
p_{n}=p_{0}+\frac{\sigma \omega}{r_{0}^{2}} \sum_{j_{0}=0}^{n-1}\left(1-\frac{2 \omega}{m r_{0}} \sum_{j_{1}=0}^{j_{0}-1} p_{j-1}\right) .
$$

Using recursively the relation

$$
\sum_{j=0}^{n-1}\left(\begin{array}{l}
j \\
k
\end{array}\right)=\left(\begin{array}{c}
n \\
k+1
\end{array}\right) \text { para } n>k
$$

which can be proof by induction hypothesis [20] and equality $j=\left(\begin{array}{l}j \\ 1\end{array}\right)$, after successive reiterations we have:

$$
\begin{aligned}
p_{n} & =p_{0} \sum_{j=0}^{\left[\frac{n}{2}\right]}\left(\begin{array}{c}
n \\
2 j
\end{array}\right)\left(-\frac{2 \sigma \omega^{2}}{m r_{0}^{3}}\right)^{j} \\
& +\frac{\sigma \omega}{r_{0}^{2}} \sum_{j=0}^{\left[\frac{n}{2}\right]}\left(\begin{array}{c}
n \\
2 j+1
\end{array}\right)\left(-\frac{2 \sigma \omega^{2}}{m r_{0}^{3}}\right)^{j} .
\end{aligned}
$$

Equation (18) gives us the linear momentum of the test particle after the $n t h$ iteration, however, it is clear that the quantity here must be a continuous variable. To recover (or approximate) the continuous picture it is convenient to do $n>>1$. Thus, for $n>>1$ the following approximation is valid 20

$$
\left(\begin{array}{l}
n \\
k
\end{array}\right) \approx \frac{n^{k}}{k !} \text { with } n>k
$$

and the equation 18 then becomes:

$$
\begin{aligned}
p_{n} & =p_{0} \sum_{j=0}^{\left[\frac{n}{2}\right]} \frac{n^{2 j}}{(2 j) !}\left(-\frac{2 \sigma \omega^{2}}{m r_{0}^{3}}\right)^{j} \\
& +\frac{\sigma \omega}{r_{0}^{2}} \sum_{j=0}^{\left[\frac{n}{2}\right]} \frac{n^{2 j+1}}{(2 j+1) !}\left(-\frac{2 \sigma \omega^{2}}{m r_{0}^{3}}\right)^{j}
\end{aligned}
$$

The two summations of the equation $(20)$ are the partial series of the hyperbolic cosine and hyperbolic sine functions, respectively. At the asymptotic limit, i.e., $n>>1$ these summations then become:

$$
p_{n}=p_{0} \cosh \left(n \omega \sqrt{\frac{2 \sigma}{m r_{0}^{3}}}\right)-\sqrt{\frac{m \sigma}{2 r_{0}}} \sinh \left(n \omega \sqrt{\frac{2 \sigma}{m r_{0}^{3}}}\right) .
$$

The equation 21 represents the momentum of the particle after time interval $n \omega$ (equation (9)). If we consider that the particle moves with velocity

$$
\bar{v}=\frac{r_{n}-r_{0}}{t_{n}-t_{0}},
$$

with $\bar{v}<c$ (where $c$ is the speed of the light), then the equation 21 becomes

$p_{n}=p_{0} \cosh \left(\left(r_{n}-r_{0}\right) \mu\right)-\sqrt{\frac{m \sigma}{2 r_{0}}} \sinh \left(\left(r_{n}-r_{0}\right) \mu\right)$.

with

$$
\mu \equiv \sqrt{\frac{2 \sigma}{\bar{v}^{2} m r_{0}^{3}}} .
$$

We are considering that the system is conservative, so we can correlate the potential energy with the linear momentum through the relation 


$$
\left(p_{n}-p_{0}\right) \bar{v}=-\left(U_{n}-U_{0}\right)
$$

Consequently

$$
\begin{aligned}
U_{n} & =-p_{n} \bar{v}=-p_{0} \bar{v} \cosh \left(\left(r_{n}-r_{0}\right) \mu\right) \\
& +\bar{v} \sqrt{\frac{m \sigma}{2 r_{0}}} \sinh \left(\left(r_{n}-r_{0}\right) \mu\right) .
\end{aligned}
$$

Rewriting the equation (26) we have

$$
\begin{aligned}
U_{n} & =\left(-\frac{p_{0} \bar{v}}{2}-\frac{\bar{v}}{2} \sqrt{\frac{m \sigma}{2 r_{0}}}\right) \mathrm{e}^{-\left(r_{n}-r_{0}\right) \mu} \\
& +\left(-\frac{p_{0} \bar{v}}{2}+\frac{\bar{v}}{2} \sqrt{\frac{m \sigma}{2 r_{0}}}\right) \mathrm{e}^{\left(r_{n}-r_{0}\right) \mu}
\end{aligned}
$$

Since $\mu$ and $r_{n}-r_{0}>0$, then the second term of equation (27), should be nil to ensure convergence of potential. Thus

$$
p_{0}=\sqrt{\frac{m \sigma}{2 r_{0}}}
$$

and

$$
U_{n}=-\xi \mathrm{e}^{-\left(r_{n}-r_{0}\right) \mu},
$$

with

$$
\xi \equiv \sqrt{\frac{m \bar{v}^{2} \sigma}{2 r_{0}}} .
$$

Defining $r_{n}-r_{0} \equiv r$ and $U n \equiv U(r)$ and by the equations (24) and (28) and using Heisenberg uncertainty principle we obtain

$$
U(r)=-\frac{\sqrt{2}}{8} \hbar c \frac{\mathrm{e}^{-\mu r}}{r}
$$

We can conclude that $g^{2}=(\sqrt{2} / 8) \hbar c \approx 0.18 \hbar c$, a value very close to the semiempirical adopted by Gauthier et al. 21] of $0.1 \hbar c$.

\section{Discussion and Conclusions}

In this work we propose an alternative iterative approach to obtain the potential of Yukawa, based in the works M. M. de Souza [17] where the classical electromagnetic field of a spinless point electron was described in a formalism with extended causality by discrete finite point-vector fields with discrete and localized point interactions (the same formalism were used to described the general relativity homogeneous field equations [18]). The intention was not to propose a better or simpler approach to the usual ones. However we were able to obtain the Yukawa Potential without directly solving the Schrödinger equation using only the Heisenberg Uncertainty principle. In summary therefore, even if the results presented here are not new from a physical point of view, the analytical method proposed here can serve as a basis for students to create their own models.

\section{5. acknowledgments}

The author would like to thank the CAPES, CAPES and $\mathrm{CNPq}$ funding agencies and the anonymous referee for their suggestions.

\section{References}

[1] H. Yukawa, Proc. Phys. Math. Sot. Japan 17, 48 (1935).

[2] C.M.G. Lattes, H. Muirhead, G.P.S. Occhialini and C.F. Powell, Nature 159, 694 (1947).

[3] J.S. Rowlinson, Physica A 156, 15 (1989).

[4] P.S. Laplace, Oeuvres de Laplace (Imprim. Roy., Paris, 1847), v. 5, p. 480.

[5] C. Neumann, Allgemeine Untersuchungen über das Newton'sche Princip der Fernwirkungen mit besonderer Rücksicht auf die elektrischen Wirkungen (Teubner, Leipzig, 1896), p. 30.

[6] J.S. Rowlinson, J. Stat. Phys. 20, 197 (1979).

[7] L.S. Ornstein and F. Zernike, Proc. Roy. Acad. Sci. Amsterdam 17, 793 (1914).

[8] L.G. Gouy, J. Phys. Theor. Appl. 9, 457 (1910).

[9] D.L. Chapman, Phil. Mag. 25, 475 (1919).

[10] C.O. Edet, P.O Okoi and S.O. Chima, Rev. Bras. Ens. Fís. 42, e20190083 (2020).

[11] U.S. Okorie, C.O Edet, A.N Ikot, G.J Rampho and R. Sever, Indian J. Phys. (2020).

[12] C.O. Edet and P.O. Okoi, Rev. Mex. Fís. 65, 333 (2019).

[13] C.O. Edet, K.O. Okorie, H. Louis and N.A. Nzeata-Ibe, Indian J. Phys. 94, 243 (2020).

[14] C.O. Edet, U.S. Okorie, A.T. Ngiangia and A.N. Ikot, Indian J. Phys. 94, 425 (2020).

[15] B.I. Ita, H. Louis, O.U. Akakuru, N.A. Nzeata-Ibe, A.I. Ikeuba, T.O. Magu, P.I. Amos and C.O. Edet, Bulg. J. Phys. 45, 323 (2018).

[16] C.N. Isonguyo, K.J. Oyewumi and O.S. Oyun, Int. J. Quantum Chem.118, e25620 (2018).

[17] M.M. de Souza, J. Phys. A: Math. Gen. 30, 6565 (1997).

[18] M.M. de Souza and R.N. Silveira, Class. Quantum Grav. 16, 619 (1999).

[19] B.R. Segatto, J.C.S. Azevedo and M.M. de Souza, J. Phys. A: Math. Gen. 36, 5115 (2003).

[20] B.R. Segatto, Rev. Bras. de Ens. Fís, 42, e20180346 (2020).

[21] N. Gauthier and S. Sherrit, Amer. J. Phys. 59, 1144 (1991). 